\title{
Stellar Populations and Kinematics in Spiral Galaxies
}

\section{Lauren A. MacArthur ${ }^{1}$, J. Jesús González ${ }^{2}$, Stéphane Courteau ${ }^{3}$ \& Michael McDonald ${ }^{4}$}

\author{
${ }^{1}$ Herzberg Institute of Astrophysics, National Research Council of Canada/University of \\ Victoria, Victoria, BC, Canada; email: Lauren.MacArthur@nrc-cnrc.gc.ca \\ ${ }^{2}$ Universidad Nacional Autónoma de México, México \\ ${ }^{3}$ Queen's University, Kingston, ON, Canada \\ ${ }^{4}$ University of Maryland, College Park, MD, USA
}

\begin{abstract}
We present a detailed study of the stellar populations (SPs) and kinematics of the bulge and inner disk regions of nearby spiral galaxies (Sa-Sd) based on deep long-slit Gemini/GMOS data. We find that the SPs of spiral galaxies are not well matched by single episodes of star formation; representative SPs must involve average SP values integrated over the star formation history (SFH) of the galaxy, such as those derived from the "full population synthesis" method used here. Our spiral bulges follow the same correlations of increasing light-weighted age and metallicity with central velocity dispersion as those of elliptical galaxies and early-type bulges found in other studies, but when SFHs more complex and realistic than a single burst are invoked, the trend with age is shallower and its scatter much reduced. In a mass-weighted context, all bulges are predominantly composed of old and metal-rich SPs. Bulge formation appears to be dominated by early processes that are common to all spheroids, whether they currently reside in disks or not. While monolithic collapse cannot be ruled out in some cases, merging must be invoked to explain the SP gradients in most bulges. Further bulge growth via secular processes, or "rejuvenated" star formation, generally contributes minimally to the stellar mass budget. We also demonstrate how the combination of our full population synthesis modeling of high-quality optical spectra of integrated SPs along with optical-NIR broad-band imaging can single out potential model weaknesses and help determine the reliability of the inferred SFHs.
\end{abstract}

Keywords. galaxies: spiral, galaxies: stellar content, galaxies: formation

\section{Introduction}

A detailed understanding of the stellar populations (SPs) that make up the integrated spectral energy distributions (SEDs) of both local and distant galaxies can provide important constraints for galaxy formation models. However, in confronting observations with SP synthesis models we are faced with a number of challenges. While SP modeling has seen tremendous progress over the past decade (e.g. Bruzual \& Charlot 2003 [BC03]; Maraston 2005 [Mar05]; Coelho et al. 2007), misinterpretations in data-model comparisons are still common.

Broadband colors are often used as a proxy for SP parameters (e.g. MacArthur et al. 2004 [Mac04]). The well-known age/ $Z$ degeneracy in optical colors is greatly lifted by the addition of NIR bands. However, in these color-based analyses, extinction and reddening effects from interstellar dust cannot be ruled out. In the meantime, full spectrum fitting techniques have proven effective at recovering the underlying stellar content of integrated galaxy spectra (e.g. Heavens et al. 2000; Cid Fernandes et al. 2005; MacArthur, González, \& Courteau 2009 [Mac09]). This type of analysis provides a stochasticallysampled SFH for a given integrated spectrum and allows for true average, as opposed 

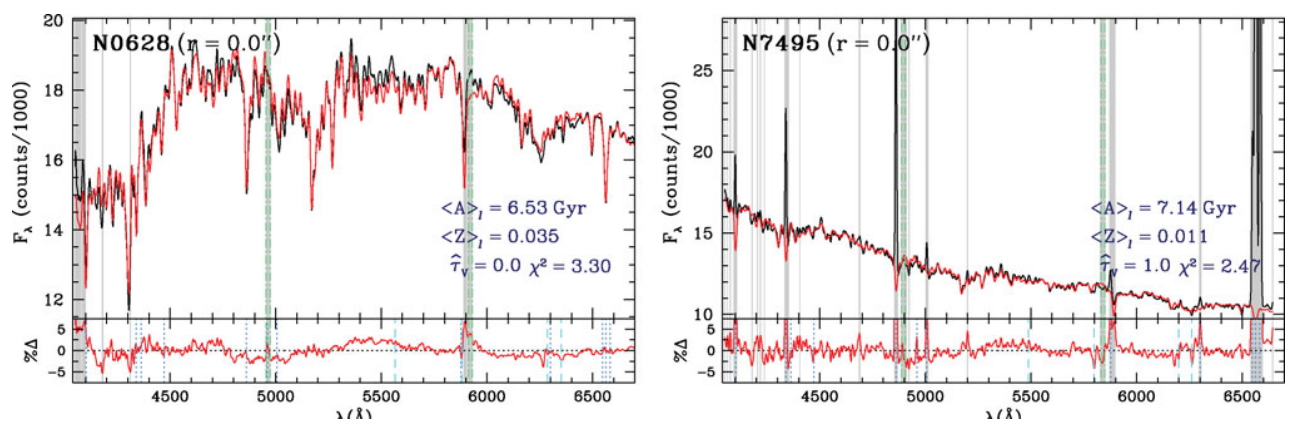

Figure 1. Full spectral synthesis fit to the central spectra of the Sc galaxies NGC 628 (left) and the emission-line dominated NGC 7495 (right). Black lines: data. Red line: model fit. Gray shading indicates emission-line regions (indicated by vertical dotted blue lines), variable night sky lines (dashed light blue lines), and CCD gaps (dashed light green line), that were excluded from the fit. Residuals are shown in the bottom panels as percent galaxy-model differences.

to single SP-based, age and $Z$ estimates. Full spectral fitting is typically limited to optical wavelengths, set by the observations. However, as most models provide SEDs in the UV-FIR range, one can use such model fits to make predictions at other wavelengths.

We provide here an overview of our full spectrum fitting technique to derive SP parameters and SFHs from integrated spectra and present results for a sample of nearby spirals (Mac09) using deep Gemini/GMOS long-slit spectra spanning 4050-6750 . We then present a comparison of these spectroscopic results with observed optical-NIR broadband colors for the same galaxies. The optical imaging $(B V R I)$ was collected at the Palomar 200-inch and the NIR ( $J H K s$ ) was obtained from the 2MASS online database.

\section{Age, Metallicity, \& SFH from Population Synthesis}

The SP fitting technique used here is the same as that described in Mac09; a brief summary is provided below. Our "full population synthesis" procedure consists of a bound constrained optimized fit representing the relative contribution of each of 70 model templates to the observed spectrum. Negative template contributions are not allowed. The templates are from the BC03 models which provide SEDs representing simple stellar populations (SSPs), i.e. single bursts of star formation (SF) at a given age and $Z$, at a resolution of $\sim 3 \AA \mathrm{FWHM} \dagger$. The selected library of 70 SSP templates cover the age range $0.001-20 \mathrm{Gyr}$ and metallicities of $Z=0.0004-0.05$ and we use the models with the Chabrier (2003) initial mass function. Our procedure also allows for dust reddening to the observed SED according to the prescription of Charlot \& Fall (2000). To accommodate any non-stellar contributions to the observed spectrum not considered in the BC03 models, we used an iterative masking scheme whereby deviant |data-model| data points are given zero weight in the fit.

Fig. 1 shows two examples of our full synthesis fits. The data-model agreement is quite remarkable throughout the entire spectrum. Analysis of the radial bins for each galaxy also demonstrated the stability of our fitting technique; indeed, Monte Carlo realizations reveal typical errors of $\ll 10 \%$ on the derived parameters.

Our study is the first to provide radially resolved spectra well into galaxy disks, allowing for gradients to be assessed. Fig. 2, left panel, shows for NGC 628 the radial profiles of average age, $Z$, and dust as determined from our full spectral synthesis fits. Of particular note is the spike to older ages just beyond $\sim 1 r_{e}$. This is precisely the signature required

$\dagger$ Although see $\S 3.4 .1$ of Mac09 for a detailed analysis of the true resolution of these models. 

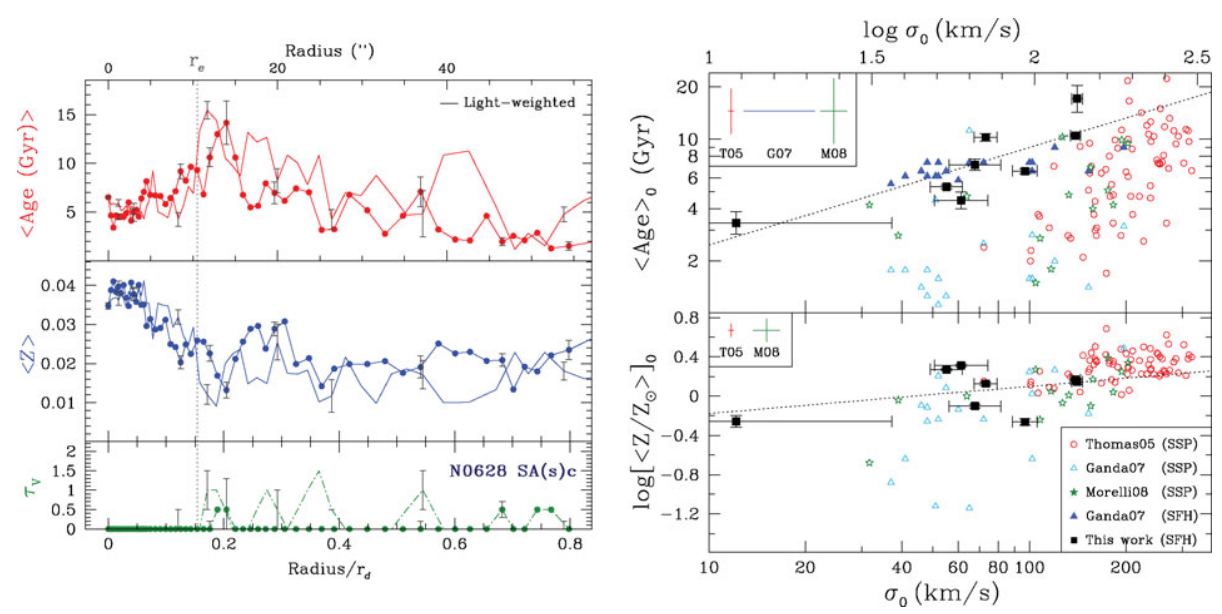

Figure 2. LEFT: Light-weighted average age (top), metallicity (middle), and dust (bottom) gradients for NGC 628. Gray error bars indicate the $1 \sigma$ confidence interval from the MC simulations. RIGHT: Average light-weighted age (top) and $Z$ (bottom) as a function of $\sigma_{0}$ compared with samples of ellipticals and bulges from the literature. Sample sources are indicated in the legend, which also indicates whether the derived parameters are SSP-based or consider a SFH.

to reconcile the discrepant results for the MW bulge noted by Thomas \& Davies (2006) where the MW bulge appears too old for its $\sigma_{0}$. This could simply be due to a positive age gradient in the inner bulge, such as observed here for NGC 628, and a sampling of the $\mathrm{SP}$ at a larger radius than the central values obtained for external galaxies.

In the right panel of Fig. 2 we plot average SP parameters versus central velocity dispersion and compare with data from the literature. The general trend of increasing age $/ Z$ with $\sigma_{0}$ are followed in all data sets, but the trend with age is much shallower and has smaller scatter when a SFH is taken into account (as opposed to SSP-based values). The implication is that bulge formation is dominated by processes that are common to all spheroids, regardless if they currently reside in a disk. The formation process occurs on timescales that decrease with increasing $\sigma_{0}$, and the relative contribution to the stellar mass budget via secular processes or "rejuvenated" star formation is small, but generally increases in weight with decreasing $\sigma_{0}$.

\section{Spectroscopy vs. Colors}

In Fig. 3 we plot the inferred SFHs from the full population synthesis fitting to the central spectra of NGC 628 \& 7495. The SFH for NGC 628 indicates a strong contribution from a 1 Gyr SSP. This is the age range where the maximum NIR excess is seen in the latest treatments of the TP-AGB stellar evolutionary phase, as shown in Fig. 3, right panel, which compares the SSP SEDs closest in age and Z of Mar05 and BC03. The presence of this 1 Gyr population can thus provide a test of the different TP-AGB treatments via comparison with the observe optical-NIR colors. In Fig. 4 we plot the colors predicted from our model fits to the observed ones for the same effective aperture. Indeed, the observed colors match much better those indicated by the Mar05 (blue arrows) than the colors from our BC03 model fits.

These results indicate that caution must be used when interpreting the optical-NIR colors of integrated stellar populations. Red colors have typically been interpreted as being due to some combination of old age, high $Z$, and significant dust extinction. However, we have shown here that in reality some of the redness can actually be due to a 

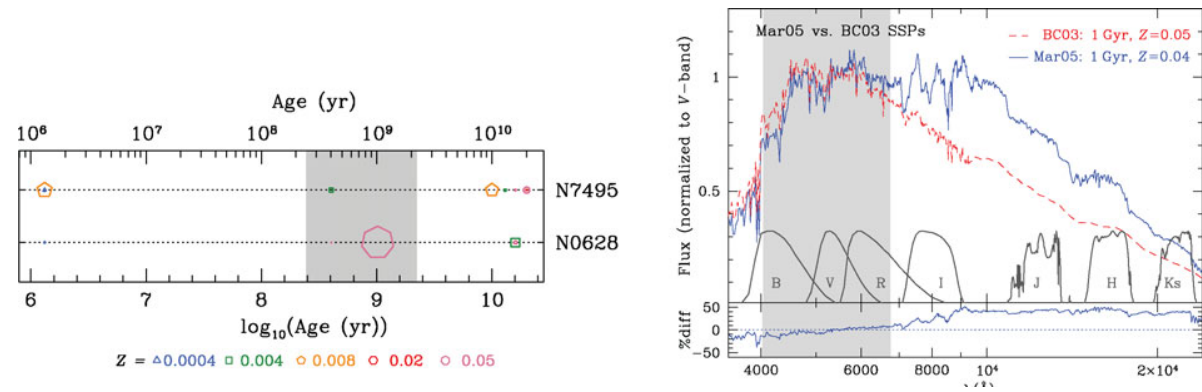

Figure 3. LEFT: Light-weighted SFHs of the central spectra for NGC 628 \& 7495 from the population synthesis fits. The dotted horizontal lines guide the eye for each galaxy (labeled at right). The horizontal axis is the SSP age. The point size is proportional to the relative weight in the fit (normalized to the $V$-band) and the colors and point types code the SSP metallicity, as indicated. The gray shading indicates the age range within which the TP-AGB phase is active. RIGHT: Comparison of SSP SEDs for the Mar05 and BC03 models. The dashed red line is the $1 \mathrm{Gyr} / Z=0.05 \mathrm{SSP}$ from the BC03 models. The solid blue line is the $1 \mathrm{Gyr} / Z=0.04 \mathrm{SSP}$ from the Mar05 models. The relevant filter response curves for our observed colors are shown in the upper panel. The lower panel plots the model differences. The gray shading represents the optical range of our GMOS spectra within which the population fits are constrained.
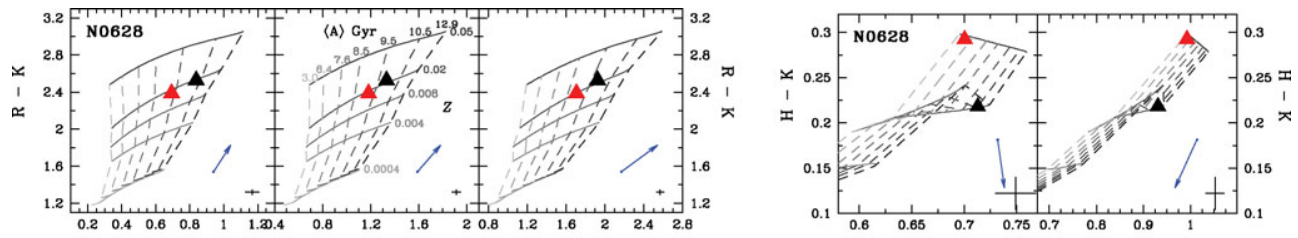

Figure 4. Color-color plots for NGC 628. The red points are colors measured ("predicted") from the BC03 model full population synthesis fits to the observed central spectrum. The black points are the corresponding observed colors. Observational errors are indicated at lower right. Overplotted are grids of BC03 models with exponential SFHs (see Mac04 for details). Solid and dashed lines are iso- $Z$ and iso-age, respectively; see left plot, middle, for labels. Blue arrows: model prediction differences between the $1 \mathrm{Gyr} / Z=0.05$ BC03 SSP (red dashed curve in Fig. 3, right panel) and the Mar05 $1 \mathrm{Gyr} / Z=0.04 \mathrm{SSP}$ (solid blue curve in Fig. 3, right panel). The arrows point toward the Mar05 predictions (from their respective location for the BC03 models).

significant contribution to the SED from a young SP with strong NIR flux excess from the TP-AGB evolutionary phase.

\section{References}

Bruzual, A. G. \& Charlot, S. 2003, MNRAS, 344, 1000 [BC03]

Chabrier, G. 2003, PASP, 115, 763

Charlot, S. \& Fall, S. M. 2000, ApJ, 539, 718

Cid Fernandes, R. et al. 2005, MNRAS, 358, 363

Coelho, P. et al. 2007, MNRAS, 382, 498

Ganda, K. et al. 2007, MNRAS, 380, 506

Heavens, A. F., Jimenez, R., \& Lahav, O. 2000, MNRAS, 317, 965

MacArthur, L. A., Courteau, S., Bell, E., \& Holtzman, J. A. 2004, ApJS, 152, 175 [Mac04]

MacArthur, L. A., González, J. J., \& Courteau, S. 2009, MNRAS, 395, 28 [Mac09]

Maraston, C. 2005, MNRAS, 362, 799

Morelli, L. et al. 2008, MNRAS, 389, 341

Thomas, D., Maraston, C., Bender, R., \& Mendes de Oliveira, C. 2005, ApJ, 621, 673

Thomas, D. \& Davies, R. L. 2006, MNRAS, 366, 510 